\section{Lupus vulgar: caso infrecuente de tuberculosis extrapulmonar}

\author{
CARMEN VARAS M. ${ }^{1}$, CONSTANZA NIEME S. ${ }^{2, a}$, CARLOS BARRÍA M. ${ }^{3}$
}

\section{Cutaneous tuberculosis. Report of one case}

Tuberculosis is uncommonly located in the skin, corresponding to 1 to $2 \%$ of extrapulmonary forms. We report a 61-year-old woman, referred due to a two months history of erythematous plaques covered with honey-colored crusts in the left preauricular region and below the chin. The lesions were previously treated as pyoderma with poor response. She was otherwise healthy, without any other symptom. Skin biopsy showed exudative tuberculoid granulomas with caseation necrosis. Koch culture was positive for Mycobacterium tuberculosis. Complementary studies ruled out other foci. Lupus vulgaris was diagnosed and antituberculous therapy started, achieving regression of cutaneous lesions.

(Rev Med Chile 2012; 140: 493-498).

Key words: Cutaneous; Lupus vulgaris; Mycobacterium infections; Tuberculosis.

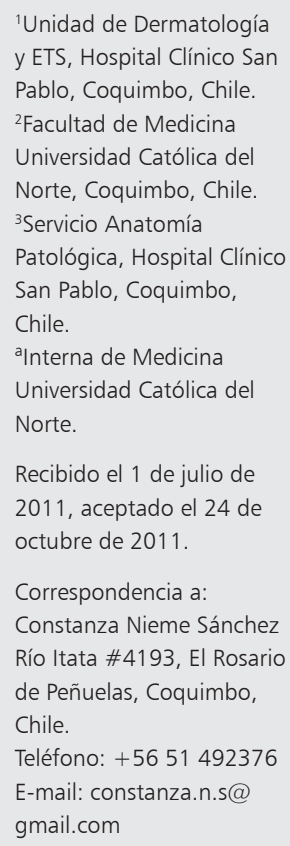

L a tuberculosis (TB) es una enfermedad infecciosa cuyo agente etiológico es $M$. El primero tiene como reservorio principal el ser humano, mientras que el segundo procede del ganado (principalmente vacuno) y sus productos derivados (carne, lácteos, etc.), desde donde se transmite al hombre ${ }^{2}$.

La TB constituye un problema de salud universal, según la Organización Mundial de la Salud un tercio de la población mundial se encuentra infectada, sólo $10 \%$ de los inmunocompetentes infectados presentan enfermedad a lo largo de su vida, sin embargo, en inmunosuprimidos la probabilidad de enfermar aumenta ${ }^{3}$. La TB cutánea es una forma inusual de presentación, se reporta con una incidencia de $0,1-1 \%$ de todos los desordenes dermatológicos ${ }^{1,4}$ y abarca entre 1-2\% de los casos de TB extrapulmonar ${ }^{1,5-7}$.

A continuación se describe caso clínico de tuberculosis cutánea en una paciente inmunocompetente y que se presentó inicialmente simulando una piodermitis. Posteriormente, se realizará revisión de la literatura actual respecto del tema, con énfasis en los aspectos clínicos.

\section{Caso clínico}

Paciente de sexo femenino de 61 años, sin antecedentes mórbidos conocidos, consultó en policlínico de dermatología por presentar lesiones en zona preauricular izquierda y submentoniana de 2 meses de evolución, de crecimiento progresivo, tratadas previamente como piodermitis con pobre resultado. La paciente no presentaba otra sintomatología. Al examen físico destacaban placas eritematosa con superficie costrosa mielicérica exudativa en las zonas antes descritas (Figura 1). Se inició terapia antibiótica oral y tópica evolucionando con resolución de la lesión costrosa superficial y persistencia de la placa eritematosa; sin embargo, al mes se produjo reactivación de las lesiones. Bajo sospecha de lupus vulgar se realizó biopsia cutánea de ambas lesiones que evidenció atrofia epidérmica, granulomas tuberculoídeos productivos y exudativos con necrosis de caseificación (Figuras 2A y B). Cultivo de Koch fue positivo para Mycobacterium tuberculosis. PPD (+) $19 \mathrm{~mm}$, radiografía de tórax normal, baciloscopias negativas (tres), resto del estudio complementario descartó otro foco TB. 
Se realizó tratamiento antituberculoso diario con isoniacida, rifampicina y pirazinamida por 2 meses y luego bisemanal con isoniacida y rifampicina por 4 meses con resolución completa de las lesiones.

\section{Clasificación de tuberculosis cutánea}

Existe un amplio espectro de formas clínicas de TB cutáneas que dependen del agente causal (número y virulencia), puerta de entrada, vía de infección y estado inmunológico del huésped ${ }^{4,5,8}$.

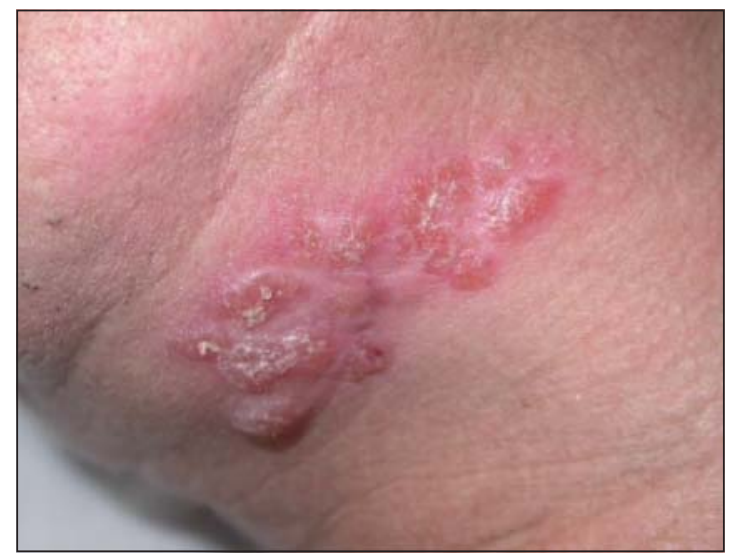

Figura 1. Caso clínico. Placa eritematosa con superficie costrosa mielicérica exudativa en región submentoneana izquierda.
La infección puede ser exógena o endógena, esta última es la más habitual. Individuos con inmunidad eficaz tienden a presentan formas localizadas, en general sin bacilos en la histopatología.

La clasificación más aceptada en la actualidad es la propuesta por Beyt ${ }^{1}$ que divide las TB cutáneas en 2 grandes grupos: TB cutáneas "verdaderas" y "tuberculides" (Tabla 1), según sea posible o no establecer el diagnóstico microbiológico respectivamente. Dividiendo los distintos subtipos según su presentación clínica y características anatomopatológicas. El lupus vulgar es la forma cutánea de TB más frecuente ${ }^{6,9}$.

\section{Lupus vulgar}

Se encuentra dentro de las TB cutáneas endógenas, causada por extensión directa de un foco tuberculoso subyacente, diseminación hematógena o linfática de un foco TB activo ${ }^{10}$. Su incidencia reportada en la literatura es muy variable, correspondiendo entre 10,9 y $63,4 \%$ de todas las TB cutáneas ${ }^{6,11-13}$. Siendo más frecuente en Europa y Asia, con cifras menores en Estados Unidos de Norteamérica y Reino Unido. No existen estudios al respecto en nuestro país.

Es una forma crónica y progresiva, afecta a todas las edades, predomina en mujeres 2-3:1, suele presentarse como lesiones únicas caracterizadas por placas irregulares de crecimiento progresivo, bien definidas, de consistencia blanda y coloración
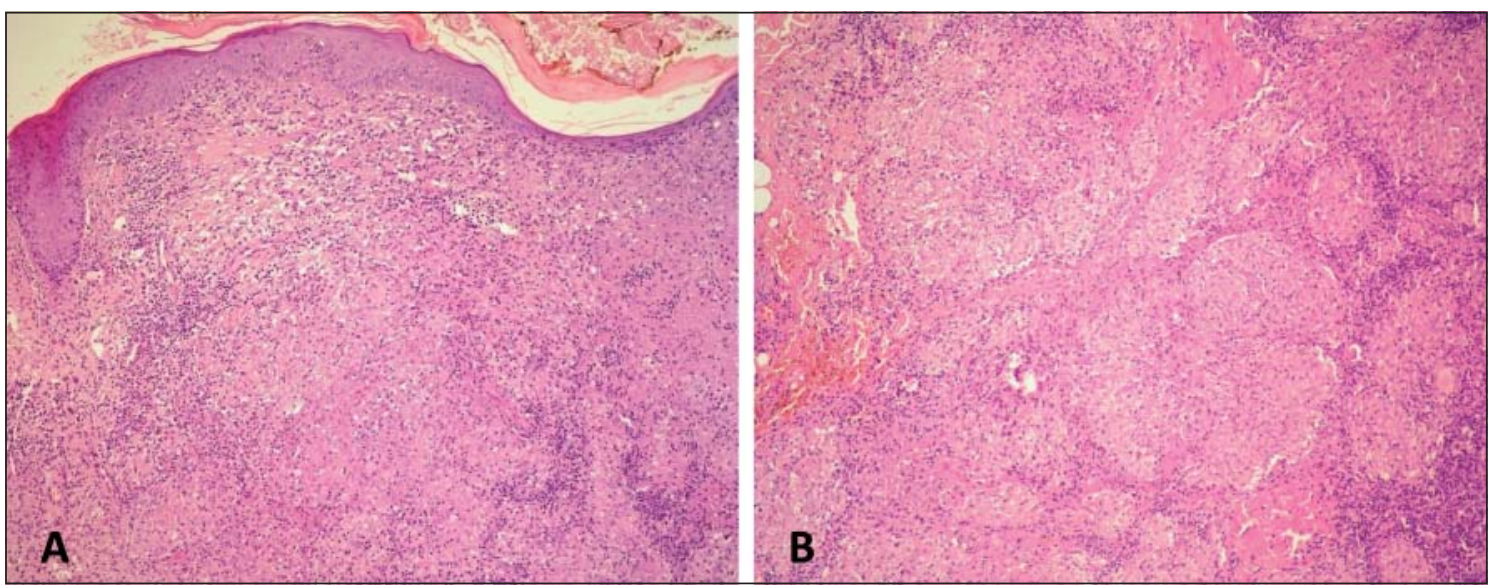

Figura 2. Histología. A. Tinción hematoxilina-eosina, aumento 10 X. Reacción granulomatosa dérmica, superficial y profunda. Epidermis exhibe leve atrofia. B. Tinción hematoxilina-eosina, aumento 20X. Granulomas tuberculoideos, productivos, epitelioides, con células gigantes y corona linfocitaria. 
Un caso de tuberculosis cutánea - C. Varas et al

Tabla 1. Clasificación de Tuberculosis cutánea

\begin{tabular}{|lll|}
\hline Verdaderas & $\begin{array}{l}\text { Infección } \\
\text { exógena }\end{array}$ & $\begin{array}{l}\text { Chancro tuberculoso } \\
\text { Tuberculosis verrucosa cutis } \\
\text { Lupus vulgar (ocasional) }\end{array}$ \\
& $\begin{array}{l}\text { Infección } \\
\text { endógena }\end{array}$ & $\begin{array}{l}\text { Lupus vulgar (mayoría) } \\
\text { Escrofuloderma } \\
\text { Tuberculosis miliar cutánea } \\
\text { Tuberculosis orificial } \\
\text { Tuberculosis gomosa }\end{array}$ \\
Tuberculides & $\begin{array}{l}\text { Eritema indurado } \\
\text { Tuberculides papulonecróticas } \\
\text { Liquen escrofulosorum } \\
\text { Flebitis nodular granulomatosa }\end{array}$ \\
\hline
\end{tabular}

pardo-rojiza ${ }^{1,4}$. Tiene varios tipos clínicos: en placa, ulcerativo y mutilante, vegetante, pseudotumoral/ hipertrófico, post-exantemático o de las membranas mucosas ${ }^{8}$. Se localiza preferentemente $(80 \%)$ en la cabeza y cuello, sobretodo en la nariz, mejillas y lóbulos auriculares ${ }^{14}$. En $40 \%$ existe linfadenitis tuberculosa asociada y en 10-20\% coexistencia con TB pulmonar u ósea ${ }^{1}$. El estudio histológico evidencia granulomas tuberculoides en la dermis superior, es frecuente encontrar alteraciones secundarias como atrofia, ulceración, acantosis e hiperplasia pseudocarcinomatosa ${ }^{15}$. El bacilo es difícil de encontrar por lo que los cultivos son generalmente negativos; sólo $6 \%$ de los cultivos son positivos ${ }^{7}$. Su principal complicación, aunque infrecuente, es el desarrollo de carcinoma espinocelular ${ }^{8}$.

\section{Diagnóstico}

El diagnóstico es difícil de establecer. Se basa en la historia, presentación clínica, histopatología compatible, prueba de tuberculina positiva y buena respuesta a la terapia específica ${ }^{10}$. A pesar que el diagnóstico definitivo de TB requiere evidencia de $M$. tuberculosis en cultivos especiales, cortes histológicos o secreciones corporales, resultados negativos no excluyen el diagnóstico ${ }^{10,16}$.

El cultivo continúa siendo el gold standard, sin embargo, el tiempo requerido para el aislamiento e identificación de la micobacteria puede tomar hasta 8 semanas ${ }^{17}$.

Recientemente, ha sido desarrollado el método de reacción en cadena de polimerasa (PCR) para la rápida identificación de M. tuberculosis, ofreciendo la posibilidad de diagnóstico el mismo día en que es tomada la muestra, incluso mediante la utilización de cantidades mínimas de tejido se obtiene un alto grado de sensibilidad y especificidad para identificar y diferenciar especies de micobacterias ${ }^{1,10,18}$. La PCR ya ha sido utilizada en la identificación de $M$. tuberculosis en lesiones de lupus vulgar, escrofuloderma, flebitis nodular granulomatosa y tuberculides papulonecróticas ${ }^{10}$.

El diagnóstico diferencial debe plantearse con sarcoidosis, leishmaniasis cutánea, linfomas, lupus discoide, lepra tuberculoide, sífilis terciaria e infección por micobacterias atípicas. A continuación se mencionan elementos útiles para orientar el diagnóstico ${ }^{1,19-21}$ :

- Sarcoidosis: enfermedad granulomatosa de causa desconocida, manifestada por múltiples lesiones maculares o papulares periorificiales en rostro, de color piel, marrón, amarillentas o violáceas, y placas infiltrativas marrón o púrpuras de forma anular, policíclica y serpentiginosa en tronco, glúteos y extremidades, con leve atrofia central. Puede asociarse a síntomas generales como fiebre y artralgias. El diagnóstico definitivo se logra a través de biopsia cutánea que muestra los característicos "tubérculos desnudos".

- Leishmaniasis cutánea: se caracteriza por pápulas en el sitio de picadura del insecto flebótomo infectado, que posteriormente evolucionan a nódulos y úlceras que cicatrizan espontáneamente a lesiones cicatriciales atróficas. Es causada por cerca de 20 especies de Leishmania (protozoo intracelular estricto). La sospecha clínica se confirma demostrando el agente en tejido obtenido por biopsia cutánea o cultivos especiales.

- Linfomas cutáneos: conjunto de trastornos linfoproliferativos que afectan primero la piel y luego el resto del sistema linforreticular. El más frecuente es la micosis fungoide, cuyas manifestaciones cutáneas se clasifican en etapa de parches, placas y etapa tumoral:

- Parches: parches eritematosos, descamativos o no, bien o mal definidos, distribuidos aleatoriamente.

- Placas: redondeadas, ovaladas, en ocasiones arciformes o anulares, de distribución aleatoria. A partir de este estadio es posible encontrar linfoadenopatias asociadas. 
- Tumores: nódulos o tumores confluentes, con o sin ulceración, que pueden asociarse a eritrodermia, queratodermia palmo-plantar, alopecia y poiquilodermia.

La biopsia cutánea con inmunohistoquímica, que en etapas iniciales es necesario realizar en forma reiterada, establece el diagnóstico.

- Lupus discoide: se presenta como placas eritematosas redondeadas u ovaladas, anulares o policíclicas, con escamas adherentes queratósicas y taponamiento folicular que pueden evolucionar a máculas hipopigmentadas o cicatrices atróficas. Es una patología crónica de etiología autoinmune que afecta exclusivamente la piel, puede precipitarse por la exposición solar, y su diagnóstico se confirma por medio de estudio histopatológico.

- Lepra tuberculoide: causada por M. leprae, infecta piel y nervios cutáneo, y se caracteriza por máculas hipoestésicas hipopigmentadas de tamaño variable, bien definidas, con bordes elevados eritemato-violáceos, área central atrófica y engrosamiento de nervios periféricos. $\mathrm{La}$ detección del agente por visualización directa o PCR confirma el diagnóstico.

- Sífilis terciaria: infección sistémica crónica provocada por Treponema pallidum. Presenta alteraciones cutáneas, vasculares y/o neurológicas. El sifiloma, aunque infrecuente, es su manifestación cutánea principal, se caracteriza por placas solitarias indoloras nodulares o papuloescamosas que pueden ulcerarse, formar círculos o arcos, expandirse rápidamente y curar sin dejar cicatriz. El diagnóstico se basa en los hallazgos clínicos y su confirmación a través de pruebas treponémicas y biopsia cutánea.

- Micobacterias atípicas: pueden presentarse como lesiones nodulares, linfocutáneas, esporotricoides o forunculosis. Su diagnóstico se basa en la detección de micobacterias distintas del complejo M. tuberculosis y M. leprae en exámenes histoquímicos y cultivos especiales.

Los hallazgos clínicos, hematológicos, histológicos y el aislamiento de $M$. tuberculosis en cultivo y/o detección de su DNA con PCR, contribuirán a esclarecer el diagnóstico ${ }^{1,4,19}$.

\section{Tratamiento}

El tratamiento de la TB cutánea debe ser el mismo que el de otras tuberculosis sistémicas ${ }^{1,8,22}$ cumpliendo con los 3 pilares básicos de la terapia; que sea asociada, controlada y prolongada, con objetivo de curar rápido las lesiones, prevenir recidivas y evitar resistencias medicamentosas. El tratamiento consta de 2 fases ${ }^{1,8}$ :

- $\quad 1^{\circ}$ Fase diaria (2 meses): Elimina la mayor cantidad de bacilos en crecimiento, se emplean 4 drogas (isoniacida, rifampicina, pirazinamida y etambutol, este último en caso de coexistencia con TB pulmonar activa).

- $2^{\circ}$ Fase bisemanal (4 meses): Esteriliza las lesiones y requiere del uso de sólo 2 drogas (isoniacida y rifampicina). Se aconseja que en pacientes portadores del virus de inmudeficiencia humana $(\mathrm{VIH})$ esta fase del tratamiento sea más prolongada ${ }^{23}$.

El régimen terapéutico planteado es suficiente en la mayoría de los casos de TB cutánea ${ }^{24}$, y la respuesta clínica debería poder apreciarse entre la $4^{\mathrm{a}}-6^{\mathrm{a}}$ semana de iniciado el tratamiento antituberculoso $^{25}$. En el caso de las TB cutáneas, la resección quirúrgica en ocasiones puede ser necesaria como complemento a la quimioterapia ${ }^{1}$.

La falta de respuesta a la terapia plantea la posibilidad de resistencia medicamentosa. Estos casos deberán ser remitidos a especialistas con experiencia en el manejo de pacientes con $\mathrm{TB}$ multiresistente ${ }^{24}$.

\section{Discusión}

Pese a los esfuerzos realizados en los últimos años a través del "Plan Regional de Control de la Tuberculosis 2006-2015”, la TB continúa siendo un problema de salud pública dada su morbimortalidad y costos asociados ${ }^{26}$. Según el Programa Nacional de Control de la Tuberculosis, la tasa total país de incidencia de TB en todas sus formas al año 2009 fue de 13,2 por 100.000 habitantes ${ }^{27}$. Si bien globalmente son cifras aceptables y coherentes con los objetivos planteados a la fecha, constituyendo uno de los países latinoamericanos con baja prevalencia de TB (al igual que Cuba, Costa Rica y Uruguay), es importante considerar las importantes diferencias encontradas a lo largo de nuestro país, con incidencias tan altas como 41,9 por 100.000 habitantes en la región de Arica y Parinacota, 27,5 en Tarapacá y 21, 5 en la región 
del Bío-Bío ${ }^{27}$. Esta extrema variabilidad es una de las características epidemiológicas relevantes de la situación actual y de los problemas que se deberán superar si se pretende avanzar hacia la eliminación de la enfermedad como problema de salud pública en plazos razonables ${ }^{28}$.

La proporción de formas extrapulmonares se relaciona inversamente con el nivel de la endemia: a medida que disminuye la tasa total de TB, aumenta la proporción de casos extrapulmonares. En el caso de Chile esta participación alcanza el 24,8\%, muy similar a la observada en años recientes en países europeos ${ }^{28,29}$.

La TB cutánea es una manifestación infrecuente del M. tuberculosis, sin embargo, ha re-emergido en aquellas partes del mundo donde la incidencia de VIH y la resistencia a drogas antituberculosas es alta ${ }^{8,26}$, presentándose con un amplio espectro de formas clínicas.

El lupus vulgar es la más frecuente de ellas ${ }^{6,9}$. Su diagnóstico representa un reto para el clínico dada la falta de técnicas diagnósticas lo suficientemente rápidas y sensibles. Incorporar nuevas herramientas de biología molecular como la PCR contribuiría al mejor control de la tuberculosis, no sólo en la implementación de diagnósticos y terapias más oportunas sino, especialmente, para estudios de epidemiología molecular ${ }^{10,30}$.

Presentamos el caso de una paciente inmunocompetente, sin factores de riesgo ni comorbilidades, con lesiones típicas de TB cutánea e histología compatible con lupus vulgar, sin otro compromiso sistémico, inicialmente manejada como piodermitis, que gracias a la sospecha clínica e inicio de terapia adecuada logró resolución completa de sus lesiones.

Dado el aumento progresivo de inmunodeficiencias adquiridas, consecuencia de la pandemia del sida, el uso de terapias inmunosupresoras y la creciente resistencia a la medicación antituberculosa $^{1,31}$, se podría esperar que en los próximos años se presente un aumento de los casos de tuberculosis extrapulmonar y sus manifestaciones cutáneas $s^{6,23,32,33}$, de ahí la importancia del abordaje de estas poblaciones vulnerables ${ }^{34}$, así como de conocer las distintas presentaciones clínicas de TB e incluirla dentro de los diagnósticos diferenciales de las patologías dermatológicas. Todo esto constituye un elemento clave en cualquier estrategia tendiente a reducir y eventualmente eliminar la TB como problema de salud pública.

\section{Referencias}

1. Fitzpatricks TB. Dermatología en medicina general. 6a Ed. Oxemberg J, Patrone U, Rondione S, Tzal K, Vaccarezza MH, Vázquez D (editores). Madrid: Editorial Médica Panamericana S.A. 2005.

2. Carapeto FJ. Tuberculosis cutánea. ¿Enfermedad emergente?. Piel 2004; 19: 63-6.

3. Tuberculosis World Health Organization. Fact sheet 104; Noviembre 2010. Disponible en: http://www.who. int/mediacentre/factsheets/fs104/en/index.html [Acceso enero de 2011].

4. Velasco PM, Dilata CJ. Tuberculosis cutánea. Piel.1999; 14: 397-410.

5. Varas $\mathrm{C}$, et al. Tuberculosis cutánea en cicatriz quirúrgica. Actas Dermosifiliogr 2003; 94 (6): 412-3.

6. Kumar B, Muralidhar S. Cutaneous tuberculosis: a twenty-year prospective study. Int J Tuberc Lung Dis 1999; 3: 494-500.

7. Fariña MC, Gegundez MI, Piqué E, Esteban J, Martín L et al. Cutaneous tuberculosis: A clinical, histopathologic, and bacteriologic study. J Am Acad Dermatol 1995; 33: 433-40.

8. Handog EB, Gabriel TG, Pineda RT. Management of cutaneous tuberculosis. Dermatol Ther 2008; 21 (3): 154-61.

9. Pomeranz MK, Orbuch P, Shupack J, Brand R. Mycobacteria and the skin. 1a Ed. Rom WM, Garay S (editors). London: Little Brown Company; 1996. p. 657-68.

10. Barbagallo J, Tager P, Ingleton R, Hirsch RJ, Weinberg JM. Cutaneous Tuberculosis: Diagnosis and Treatment. Am J Clin Dermatol 2002; 3 (5): 319-28.

11. Ramesh V, Misra R, Beena K, Mukherjee A. A study of cutaneous tuberculosis in children. Pediatr Dermatol 1999; 16 (4): 264-9.

12. Terranova M, Padovese V, Fornari U, Morrone A. Clinical and epidemiological study of cutaneous tuberculosis in Northern Ethiopia. Dermatology 2008; 217 (1): 89-93.

13. Vashisht P, Sahoo B, Khurana N, Reddy BS. Cutaneous tuberculosis in children and adolescents: a clinicohistological study. J Eur Acad Dermatol Venereol 2007; 21: 40-7.

14. García-Rodríguez JF, Monteagudo-Sánchez B, MariñoCallejo A. Tuberculosis cutánea: estudio descriptivo de 15 años. Enferm Infecc Microbiol Clin 2008; 26 (4): 205-11.

15. Lever WF, Schaumburg-Lever G. Histopathology of the skin. Lever WF, Schaumburg-Lever G (editors). 7th Ed. Philadelphia: Lippincott, 1990: 326-33.

16. Hsiao PF, Tzen CY, Chen HC, Su HY. Polymerase chain reaction based detection of Mycobacterium tubercu- 
losis in tissues showing granulomatous inflammation without demonstrable acid-fast bacilli. Int J Dermatol 2003; 42: 281-6.

17. Kent PT, Kubica GP. Public Health Mycobacteriology: A guide for the level III Laboratory. Atlanta, GA: Centers for Disease Control, US Department of Health and Human Services; 1985.

18. Klatser PR. Amplification reactions in mycobacteriology. J Microbiol. Methods 1995; 23: 75-87.

19. Burns T, Breathnach S, Cox N, Griffiths C (editors). Rook's Textbook of Dermatology. 8th Ed. Oxford, UK: Wiley-Blackwell; 2010.

20. James WD, Berger T, Elston D. Andrews' Diseases of the Skin: Clinical Dermatology. 10th Ed. Philadelphia, PA: Saunders/Elsevier Publishers; 2006.

21. Weller R, Hunter J, Savin J and Dahl M. Clinical Dermatology. 4th Ed. Malden, Massachusetts, USA: Blackwell Publishing; 2008.

22. Izumi AK, Matsunaga J. BCG vaccine-induced lupus vulgaris. Arch Dermatol 1982; 118: 171-2.

23. Pérez B, Pifarre R, de Vera CV, García JM, Baradad M, Vilá M, Egido R. Cutaneous tuberculosis caused by Mycobacterium tuberculosis, an uncommon pathology. An Med Interna 2006; 23 (11): 560-1.

24. Bravo F, Gotuzzo E. Cutaneous tuberculosis. Clin Dermatol 2007; 25: 173-80.

25. Ramam M, Mittal R, Ramesh V. How soon does cutaneous tuberculosis respond to treatment? Implications for a therapeutic test of diagnosis. Int J Dermatol 2005; 44: 121-4.

26. Organización Panamericana de la Salud. Plan regional de tuberculosis 2006 - 2015. Washington DC: OPS; 2006.
Disponible en: http://www.paho.org/Spanish/AD/DPC/ CD/tb-reg-plan-2006-15.pdf [Acceso mayo de 2011].

27. Instituto Nacional de Estadística. Tasa de Incidencia de Tuberculosis en todas sus formas, por regiones. Chile 2000-2009. Disponible en: http://epi.minsal.cl/epi/ html/AtlasInteractivos/Nacionales/AtlasTBC/atlas.html [Acceso mayo de 2011].

28. Ministerio de Salud, Gobierno de Chile. Programa Nacional de Control de la Tuberculosis. Manual de Organización y Normas Técnicas 2005.

29. Zúñiga M, Marcone P, Riquelme C. Reflexiones sobre la tuberculosis en Chile, 2006. Rev Chil Enf Respir 2007; 23: 59-66.

30. Marcone P. Aplicaciones de la biología molecular en el Programa Nacional de Tuberculosis en Chile: ¿Lujo o necesidad?. Rev Chil Enf Respir 2006; 22: 201-8.

31. European Center for Disease Prevention and Control. Framework Action Plan to fight Tuberculosis in the European Union. Stockholm, February 2008. Disponible en: http://ecdc.europa.eu/en/publications/Publications/0803_SPR_TB_Action_plan.pdf [Acceso mayo de 2011].

32. Hayward AC, Darton T, Van-Tam JN, Watson JM, Coker $\mathrm{R}$, Schwoebel V. Epidemiology and control of tuberculosis in Western European cities. Int J Tuberc Lung Dis 2003; 7: 751-7.

33. Monteagudo B, García-Rodríguez J, León-Muiños E, Mariño A. Incidencia de tuberculosis cutánea en pacientes con otro tipo de tuberculosis. Piel 2007; 22 (6): 267-70.

34. Farga V. Hacia la erradicación de la tuberculosis. Rev Chil Enf Respir 2006; 22: 55-67. 